\title{
Técnica de Substituição Homofônica Perfeita Símbolo-a-Símbolo
}

\author{
Danielle P. B. de A. Camara e Valdemar C. da Rocha Jr.
}

\begin{abstract}
Resumo-Este artigo apresenta uma nova técnica de substituição homofônica perfeita, pertencente a uma classe denominada Substituição Homofônica Símbolo-a-Símbolo (SH-SAS). A técnica SH-SAS tem apresentado desempenho semelhante à técnica JKM modificada, alcançando a mesma eficiência, e possui a vantagem prática de utilizar sempre um único símbolo mudo.

Palavras-Chave-Teoria da informação, substituição homofônica, criptografia.

Abstract-This article presents a new perfect homophonic substitution scheme which belongs to a class named Letter by Letter (LBL) homophonic substitution. This technique has shown a performance similar to that of the modified JKM homophonic substitution scheme, reaching the same efficiency, and has the practical advantage of always employing a single dummy symbol.
\end{abstract}

Keywords-Information theory, homophonic substitution, cryptography.

\section{INTRODUÇÃO}

Historicamente alguns sistemas criptográficos de chave secreta foram quebrados [1] explorando o fato da estatística do texto claro desviar-se daquela de uma seqüência de símbolos estatisticamente independentes e uniformemente distribuídos, usualmente chamada de sequiência verdadeiramente aleatória. A substituição (ou codificação) homofônica é uma técnica antiga utilizada para converter o texto claro em uma seqüência de símbolos (mais) aleatória. Esta técnica consiste na substituição (one-to-many) de cada letra da mensagem original por um substituto ou homofonema pertencente a um alfabeto maior, com o intuito de formar o novo texto claro que é então cifrado. Cada homofonema é então codificado de forma a produzir símbolos uniformemente distribuídos e estatisticamente independentes. Tal técnica torna um criptosistema simétrico não-expansível [1] mais seguro, uma vez que aumenta a distância de unicidade da cifra [2], porém isso tem um custo: a expansão do texto claro. Em 1988, Günther [3] fez uma importante contribuição na área introduzindo o que foi denominado substituição homofônica de comprimento variável. Tanto a substituição homofônica clássica como a de comprimento variável foram abordadas em [1] em termos de teoria da informação.

Este artigo aborda uma técnica de substituição homofônica padrão pertencente a uma nova classe denominada Substituição Homofônica Símbolo-a-Símbolo (SH-SAS). Esta técnica mostrou desempenho semelhante à técnica JKM

Danielle P. B. de A. Camara e Valdemar C. da Rocha Jr., Grupo de Pesquisa em Comunicações - CODEC, Departamento de Eletrônica e Sistemas, Universidade Federal de Pernambuco, Caixa Postal 7800, 50711-970, Recife, PE, Brasil, Emails: dpbac@ieee.org, vcr@ufpe.br. modificada [4], ao se considerar a eficiência $\eta$, com o diferencial de sempre utilizar apenas um símbolo mudo, o que nem sempre ocorre quando se utiliza a técnica JKM modificada.

Na Seção II são apresentados alguns conceitos básicos, assim como algumas definições que se mostram úteis na comparação de técnicas de substituição homofônica, facilitando assim o entendimento das seções seguintes, e conseqüentemente do trabalho aqui apresentado.

Na Seção III é introduzida uma nova técnica de substituição homofônica denominada Substituição Homofônica Símboloa-Símbolo (SH-SAS). Como será visto, tal técnica apresenta eficiência $(\eta)$ igual ou, em alguns casos, maior que a obtida com a técnica Rocha-Massey (RM) [7] e desempenho semelhante à técnica JKM modificada [4]. A técnica SHSAS possui um diferencial positivo em relação à técnica JKM modificada, pelo fato de sempre usar um único símbolo mudo, enquanto que esta última necessita, em alguns casos, de mais de um símbolo mudo. A utilização de um único símbolo mudo na técnica SH-SAS é garantida pela construção descrita na Seção III. A Seção IV ilustra o uso da técnica aqui introduzida a partir de um exemplo. Por fim a Seção V encerra o artigo com algumas conclusões.

\section{Alguns CONCEITOS BÁsicos}

Seja $U$ uma fonte $K$-ária discreta sem memória (DMS) com alfabeto $\left\{u_{1}, u_{2}, \ldots, u_{K}\right\}$ e entropia

$$
H(U)=-\sum_{i=1}^{K} P_{U}\left(u_{i}\right) \log P_{U}\left(u_{i}\right) .
$$

Os símbolos que saem da fonte $U_{1}, U_{2}, \ldots$, são codificados numa seqüência de símbolos $D$-ários, $X_{1}, X_{2}, \ldots$ Assume-se neste artigo, para simplificação do tratamento, a codificação homofônica binária de uma seqüência $U_{1}, U_{2}, \ldots K$-ária, com símbolos estatisticamente independentes e identicamente distribuídos, reduzindo assim o problema de codificação da fonte de mensagem ao problema de codificação de uma única variável aleatória $U=U_{1}$. Lembrando que a teoria descrita é passível de modificação para que sejam consideradas fontes discretas com memória, bastando para isso substituir a distribuição de probabilidade de $U_{i}$ pela distribuição de probabilidade condicional de $U_{i}$ dados os valores observados $U_{1}, U_{2}, \ldots, U_{i-1}$. Além disso, admite-se que $U$ tem uma distribuição de probabilidade racional na qual $P_{U}\left(u_{i}\right)=$ $m_{i} / n_{i}, 1 \leq i \leq K$, em que $m_{i}$ e $n_{i}$ são números inteiros positivos e primos entre si.

O canal homofônico é um canal sem memória cujo alfabeto de entrada $\left\{u_{1}, u_{2}, \ldots, u_{K}\right\}$ coincide com o conjunto de possíveis valores de $U$, o alfabeto de saída $V$ pode ser finito ou 
infinito contável, e as probabilidades de transição $P_{V \mid U}\left(v_{i j} \mid u_{i}\right)$ têm a propriedade que para cada $j$ existe exatamente um $i$ tal que $P_{V \mid U}\left(v_{i j} \mid u_{i}\right) \neq 0$, observa-se desta forma que $H(U \mid V)=0$. Em geral, os $v_{i j}$ para os quais $P_{V \mid U}\left(v_{i j} \mid u_{i}\right)>0$ serão considerados homofonemas de $u_{i}$.

Um codificador $D$-ário livre de prefixo é um mecanismo que associa uma sequiência $D$-ária a cada $v_{i j}$, de modo que a palavra-código associada seja distinta das outras palavrascódigo e também não seja prefixo de outra palavra-código mais longa, o que garante que em uma seqüência de palavrascódigo o fim de cada palavra-código possa ser identificado imediatamente sem que seja necessária a verificação de quaisquer outros símbolos na sequiência. $\mathrm{O}$ comprimento médio de uma variável aleatória $W$ é denotada por $E(W)$ na qual $W$ é o comprimento do palavra-código que representa o símbolo. Dado que $l_{j}$ é o comprimento médio da palavracódigo associada ao símbolo homofonema $v_{i j}$,

$$
E(W)=\sum_{j} l_{j} P_{V}\left(v_{i j}\right) .
$$

A expansão de texto claro foi definida anteriormente [1] como o comprimento médio do homofonema menos a entropia da fonte, i.e., $E(W)-H(U)$, sendo assumido implicitamente que $H(U \mid V)=0$. Esta definição de expansão de texto claro é útil ao se comparar dois sistemas de codificação homofônica que produzem o mesmo número de bits por símbolo na saída do canal homofônico e possivelmente possuem valores distintos para $E(W)$.

No contexto de codificação de fonte, para uma dada fonte sem memória $U$ e um código unicamente decodificável associado a ela, a eficiência do código $\eta$ é definida [5, p.86] como a razão entre a entropia da fonte $H(U)$ e o comprimento médio da palavra-código $E(W)$, i.e., $\eta=H(U) / E(W)$ e como conseqüência, a redundância $\rho$ é definida como $\rho=1-\eta$, i.e. $\rho=[E(W)-H(U)] / E(W)$. A seguir são revistas as definições de redundância e de eficiência em sistemas de substituição homofônica, introduzidas em [6], denotando por $R$ a taxa de transmissão de informação do referido sistema, i.e., denotando por $R$ o número de bits por símbolo na saída de um canal homofônico.

Definição 1: A redundância $\rho$ de uma técnica de codificação homofônica é definida como a razão entre a expansão do texto claro $E(W)-R$ e o comprimento médio de um homofonema $E(W)$, i.e.,

$$
\rho=[E(W)-R] / E(W)=1-R / E(W) .
$$

Definição 2: A eficiência $\eta$ de uma técnica de codificação homofônica é definida como

$$
\eta=1-\rho=R / E(W) .
$$

Jendal-Kuhn-Massey (JKM) [1] definiram codificação homofônica como perfeita se a nova seqüência de texto claro é não-redundante e como ótima se ela além de perfeita minimizar a expansão de texto claro. Ao se comparar uma codificação homofônica para fontes distintas nota-se que uma menor expansão de texto claro não resulta necessariamente numa redundância menor, o que será ilustrado no exemplo a seguir.
Exemplo 1: Uma técnica de codificação homofônica com taxa $R_{1}=8$ e comprimento médio $E\left(W_{1}\right)=10$ tem uma expansão de texto claro $E\left(W_{1}\right)-R_{1}=2$ e uma redundância $\left[E\left(W_{1}\right)-R_{1}\right] / E\left(W_{1}\right)=0,2$, enquanto que uma técnica de codificação homofônica com taxa $R_{2}=3$ e comprimento médio $E\left(W_{2}\right)=4$ tem uma expansão de texto claro $E\left(W_{2}\right)-$ $R_{2}=1$ e uma redundância $\left[E\left(W_{2}\right)-R_{2}\right] / E\left(W_{2}\right)=0,25$.

Uma redundância menor significa mais bits de entropia por homofonema (dígito de código homofônico binário) enquanto que uma expansão de texto claro menor por se só não fornece uma interpretação objetiva. Este fato ilustra a relevância da nova definição de redundância (Definição 1) na comparação de técnicas de codificação homofônica para fontes distintas [6].

À luz destas definições de redundância e eficiência, em [6] foi também introduzida uma nova definição de uma técnica de codificação homofônica ótima.

Definição 3: Uma técnica de substituição (codificação) homofônica é definida como ótima se ela é perfeita e sua redundância é a menor possível.

\section{TÉCNICA DE SubstituiçÃo HomofônicA SÍMBOLO-A-SÍMBOLO}

Considere mais uma vez, uma fonte $U$ discreta sem memória tal que sua distribuição de probabilidade é formada por números racionais $P_{U}\left(u_{i}\right)=m_{i} / n_{i}, 1 \leq i \leq K$, em que $m_{i}$ e $n_{i}$ são números inteiros positivos e primos entre si. Se $n_{i}$ for uma potência $D$-ária, i.e., $n_{i}=D^{l_{i}}$, a técnica JKM [1], opera com cota superior finita requerendo um número finito de experimentos a fim de selecionar um homofonema associado ao elemento $u_{i}$ da fonte $U$, o que não ocorre no caso em que $n_{i} \neq D^{l_{i}}$. Caso este de interesse, já tendo sido abordado anteriormente, como por exemplo em [7], [8].

O objetivo desta seção é introduzir uma nova técnica de substituição homofônica padrão que mostra desempenho equivalente ao obtido utilizando a técnica JKM [1], considerando a eficiência $\eta$ (Definição 2) como parâmetro de comparação.

De modo similar à técnica JKM modificada [4], na técnica SH-SAS, cada palavra-código homofônica é construída como a concatenação de palavras-código mais curtas derivadas a partir das probabilidades da fonte, resolvendo desta forma o problema de ter que armazenar um número infinito contável de palavras-código homofônicas, no caso em que $n_{i}$ não é uma potência de $D$.

Observa-se que diferentemente da técnica JKM modificada, na técnica SH-SAS sempre se utiliza apenas um símbolo, i.e., independente da expansão do símbolo da fonte apenas uma palavra-código será associada ao símbolo mudo, o que é garantido pela construção descrita a seguir, sendo ilustrado no Exemplo 2 da Seção IV.

$\mathrm{Na}$ técnica SH-SAS, a fonte é requisitada a emitir um símbolo $u_{i}$ e a partir daí um procedimento é feito a fim de determinar o homofonema associado a este símbolo. Tal procedimento é descrito a seguir.

1) Descrição da técnica SH-SAS: Com a finalidade de simplificar a descrição, é considerado o caso binário, i.e., $D=2$. 
a) Para cada símbolo da fonte $U=u_{i}, 1 \leq i \leq K$, com probabilidade $P_{U}\left(u_{i}\right)=m_{i} / 2^{s_{i}}$, i.e., para o qual $n_{i}=2^{s_{i}}$, em que $s_{i}$ é um inteiro positivo, escreve-se $m_{i}$ na base 2 e a cada termo desta decomposição em base 2 associa-se um homofonema, tal que $P_{V}\left(v_{i j}\right)=2^{-l_{j}}$.

b) Para cada símbolo da fonte $U=u_{i}, 1 \leq i \leq K$, com probabilidade $P_{U}\left(u_{i}\right)=m_{i} / n_{i}$, para o qual $n_{i} \neq 2^{s_{i}}$, associam-se dois tipos de símbolos chamados, respectivamente, símbolo homofonema e homofonema mudo.

Considere que $n$ é o menor denominador comum dessas probabilidades. Sendo $n=2^{r} n^{\prime}$ em que $n^{\prime}$ é o produto dos fatores ímpares de $n$ e $r$ um inteiro positivo, escolha o menor inteiro positivo $s$ tal que $n^{\prime} \mid\left(2^{s}-1\right)$. A probabilidade do homofonema mudo, $\Delta$, é dada por $1 / 2^{s}$.

1. A decomposição da probabilidade de um ou mais símbolos $u_{i}$ da fonte possui apenas termos periódicos. Dado que a fonte $U$ seleciona um desses símbolos, um experimento é feito em que o símbolo mudo tem probabilidade $P(\Delta)=P\left(\Delta \mid u_{i}\right)=1 / 2^{s}$, e desta forma o $j$-ésimo homofonema, denotado por $v_{i j}$, associado a $u_{i}$ é selecionado com probabilidade

$$
P_{V \mid U}\left(v_{i j} \mid u_{i}\right)=P_{V}\left(v_{i j}\right) / P_{U}\left(u_{i}\right),
$$

observando que $P_{V}\left(v_{i j}\right)$ é o j-ésimo termo da decomposição em potências negativas de 2 de $\frac{m_{i} \cdot d}{2^{s}}$, em que $d$ é um número inteiro positivo e

$$
P_{U}\left(u_{i}\right)\left(1-2^{-s}\right)=\left(\frac{2^{s}-1}{2^{s}}\right)\left(\frac{m_{i}}{n_{i}}\right)=\frac{m_{i} \cdot d}{2^{s}} .
$$

Assim $V$ é uma fonte discreta sem memória tendo como símbolos os símbolos homofonemas de $U$ com probabilidade

$$
P_{V}\left(v_{i j}\right)=P_{U}\left(u_{i}\right) P_{V \mid U}\left(v_{i j} \mid u_{i}\right)
$$

e um homofonema mudo com probabilidade $P(\Delta)=$ $P\left(\Delta \mid u_{i}\right)=1 / 2^{s}$.

2. A decomposição da probabilidade de um ou mais símbolos da fonte possui termos não-periódicos, em que a probabilidade do $j$-ésimo termo não-periódico associado ao símbolo $u_{i}$ é

$$
P_{V}\left(v_{i j}\right)=2^{-l_{j}},
$$

na qual $l_{j}$ é um número inteiro positivo. A probabilidade de seleção deste termo é

$$
P_{V \mid U}\left(v_{i j} \mid u_{i}\right)=2^{-l_{j}} / P_{U}\left(u_{i}\right)
$$

e caso este termo seja selecionado, seu respectivo homofonema será gerado, sem necessidade de considerar o símbolo mudo. A soma das probabilidades dos homofonemas associados aos termos não-periódicos é portanto $\sum_{t} 2^{-l_{t}}$. Então os homofonemas $v_{i j}$ associados aos termos periódicos são selecionados com probabilidade

$$
P_{V \mid U}\left(v_{i j} \mid u_{i}^{\prime}\right)=\frac{P_{V}\left(v_{i j}\right)}{P_{U}\left(u_{i}\right)-\sum_{t} 2^{-l_{t}}},
$$

na qual fizemos uso de um símbolo virtual $u_{i}^{\prime}$, ocorrendo com probabilidade $P_{U}\left(u_{i}\right)-\sum_{t} 2^{-l_{t}}$ e observando que $P_{V}\left(v_{i j}\right)$ é o $j$-ésimo termo da decomposição em potências negativas de 2 de

$$
\left[P_{U}\left(u_{i}\right)-\sum_{t} 2^{-l_{t}}\right] \cdot\left[1-2^{-s}\right],
$$

lembrando que o símbolo mudo possui probabilidade $2^{-s}$.

Se o termo não-periódico não for tratado desta forma, i.e., um termo que ao ser selecionado é imediatamente relacionado a um homofonema, aparecerão termos repetidos na representação do símbolo da fonte, e como é mostrado em [1], ao se considerar a mesma fonte, se a técnica de substituição homofônica é ótima então a decomposição de $P_{U}\left(u_{i}\right)$ para cada $u_{i}$ deve consistir de potências negativas de 2 distintas, ou seja, considerando a expansão em termos distintos, $E(W)$ é minimizado e portanto $\eta$ é maximizada para tal fonte. Tal situação é ilustrada na forma de exemplo (Exemplo 2).

c) A codificação binária nesta técnica é feita da seguinte forma.

1. Inicia-se uma árvore binária associando-se às folhas distantes da raiz $l_{j}$ ramos os homofonemas cuja probabilidade é igual a $P_{V}\left(v_{i j}\right)=P_{U}\left(u_{i}\right) P_{V \mid U}\left(v_{i j} \mid u_{i}\right)=2^{-l_{j}}$. Note que a probabilidade do símbolo mudo é a probabilidade de transição $P\left(\Delta \mid u_{i}\right)$ e, portanto nesta técnica poderá estar associada a uma palavra-código terminada num nó intermediário da árvore.

2. As palavras-código são construídas associando aos ramos superiores " 0 " e inferiores "1", seguindo então, o caminho da raiz à folha associada ao homofonema.

3. A construção da palavra-código associada ao símbolo mudo é feita de forma similar à construção da palavracódigo do homofonema, seguindo na árvore um percurso que vai até o nó ou folha associada ao $\Delta$.

Para evitar ambigüidade na decodificação deve-se observar que a palavra-código formada pela concatenação do símbolo mudo e de um homofonema não deve ser igual a outra palavra-código homofônica, nem prefixo de uma outra palavra-código homofônica mais longa. Com exceção das palavras-código associadas a homofonemas que não fazem uso do símbolo mudo, uma vez que nesse caso o símbolo mudo nunca precederá tal palavracódigo homofônica.

Desta forma tal código é um código unicamente decodificável, tornando a decodificação trivial como será visto mais adiante, no item e).

d) Quando um homofonema mudo $\Delta$ é produzido no passo b), um símbolo mudo $\Delta$ é produzido por $V$ e o experimento de seleção é repetido quantas vezes forem necessárias até que o correspondente símbolo homofonema $v_{i j}$, seja selecionado, tendo como resultado uma seqüência do tipo $\Delta \Delta \Delta \Delta \ldots \Delta v_{i j}$. A fonte $U$ é então requisitada a selecionar o próximo símbolo a ser codificado e assim por diante.

e) A decodificação é imediata, bastando apenas apagar as 
palavras-código que representam os símbolos mudos e, em seguida, mapear as palavras-código restantes aos correspondentes símbolos de $U$.

A seguir a técnica aqui introduzida é ilustrada e comentada por meio de um exemplo.

\section{EXEMPlo Ilustrativo}

Nesta seção é apresentado um exemplo onde são aplicadas as técnicas de substituição homofônica SH-SAS, JKM modificada [4] e Rocha-Massey (RM) [7].

Exemplo 2: Considere uma fonte discreta binária sem memória com $K=3$ e obedecendo à seguinte distribuição de probabilidade $P_{U}\left(u_{1}\right)=3 / 10, P_{U}\left(u_{2}\right)=5 / 12$ e $P_{U}\left(u_{3}\right)=$ $17 / 60$.

Segundo o passo b) da descrição da técnica SH-SAS, $n=$ $2^{2} \cdot 3 \cdot 5=60$, logo, $n^{\prime}=3 \cdot 5=15$ e $s=4$, portanto $P(\Delta)=P\left(\Delta \mid u_{i}\right)=1 / 16$.

Procedendo a decomposição das probabilidades dos símbolos da fonte, usando a técnica em [9] obtém-se,

$$
\begin{gathered}
P_{U}\left(u_{1}\right)=\frac{3}{10}=\left(\frac{1}{4}\right) \sum_{i=0}^{\infty}\left(\frac{1}{16}\right)^{i}+\left(\frac{1}{32}\right) \sum_{i=0}^{\infty}\left(\frac{1}{16}\right)^{i}, \\
P_{U}\left(u_{2}\right)=\frac{5}{12}=\frac{1}{4}+\left(\frac{1}{8}\right) \sum_{i=0}^{\infty}\left(\frac{1}{4}\right)^{i} \\
P_{U}\left(u_{3}\right)=\frac{17}{60}=\frac{1}{4}+\left(\frac{1}{32}\right) \sum_{i=0}^{\infty}\left(\frac{1}{16}\right)^{i}
\end{gathered}
$$

A decomposição de $P_{U}\left(u_{1}\right)$ possui apenas termos periódicos e, portanto, pelo procedimento descrito em 1 . de b) da Seção III-.1 resulta

$$
P_{U}\left(u_{1}\right) \cdot(1-1 / 16)=(3 / 10) \cdot(15 / 16)=1 / 4+1 / 32 .
$$

Usando (5), as probabilidades de escolha dos homofonemas $v_{11}$ e $v_{12}$ associados ao símbolo $u_{1}$ são:

$$
P_{V \mid U}\left(v_{11} \mid u_{1}\right)=(1 / 4) /(3 / 10)=5 / 6
$$

$\mathrm{e}$

$$
P_{V \mid U}\left(v_{12} \mid u_{1}\right)=(1 / 32) /(3 / 10)=5 / 48 .
$$

As probabilidades dos homofonemas $v_{11}$ e $v_{12}$ são, respectivamente, $P_{V}\left(v_{11}\right)=1 / 4$ e $P_{V}\left(v_{12}\right)=1 / 32$ (Vide Equação (7)).

Já as probabilidades dos símbolos $u_{2}$ e $u_{3}, P_{U}\left(u_{2}\right)=$ $\frac{5}{12}$ e $P_{U}\left(u_{3}\right)=\frac{17}{60}$, respectivamente, apresentam em suas decomposições termos não-periódicos, desta forma é seguido o procedimento descrito em 2. de b) da Seção III-.1.

De (9) observa-se que os termos não-periódicos associados a $u_{2}$ e $u_{3}$, são selecionados com probabilidades,

$$
P_{V \mid U}\left(v_{21} \mid u_{2}\right)=(1 / 4) /(5 / 12)=3 / 5
$$

$\mathrm{e}$

$$
P_{V \mid U}\left(v_{31} \mid u_{3}\right)=(1 / 4) /(17 / 60)=15 / 17,
$$

respectivamente. Observe que aqui não há necessidade de escolha entre símbolo homofonema e homofonema mudo.

Os demais homofonemas associados ao símbolo $u_{2}$ são selecionados com probabilidades (Vide Equação (10)),

$$
P_{V \mid U}\left(v_{22} \mid u_{2}^{\prime}\right)=3 / 4
$$

e

$$
P_{V \mid U}\left(v_{23} \mid u_{2}^{\prime}\right)=3 / 16
$$

uma vez que por (11) tem-se $[5 / 12-1 / 4] \cdot[1-1 / 16]=\frac{5}{32}=$ $\frac{1}{8}+\frac{1}{32}$ e, portanto, as probabilidades dos homofonemas $v_{22}$ e $v_{23}$ associados a $u_{2}$ são, respectivamente, $P_{V}\left(v_{22}\right)=1 / 8 \mathrm{e}$ $P_{V}\left(v_{23}\right)=1 / 32$.

De modo similar, o homofonema $v_{32}$ associado ao símbolo $u_{3}$ é selecionado com probabilidade (Vide Equação 9),

$$
P_{V \mid U}\left(v_{32} \mid u_{3}^{\prime}\right)=15 / 16,
$$

uma vez que por $(11)$, tem-se $[17 / 60-1 / 4] \cdot[1-1 / 16]=$ $1 / 32$, e desta forma a probabilidade do homofonema $v_{32}$ associado ao símbolo $u_{3}$ é $P_{V}\left(v_{32}\right)=1 / 32$. O resultado é ilustrado na Figura 1.

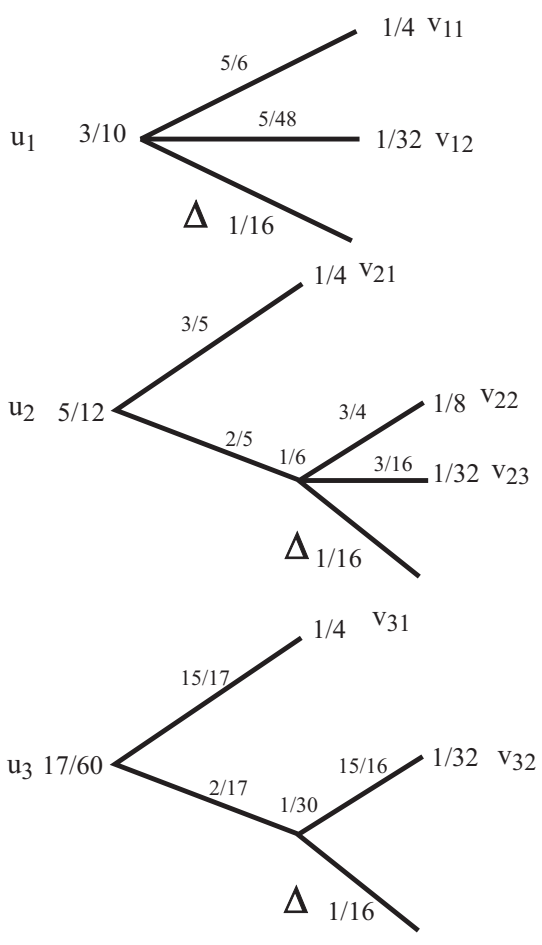

Fig. 1. Nova técnica de substituição homofônica símbolo-a-símbolo para a fonte $U$ com distribuição de probabilidade $P_{U}\left(u_{1}\right)=3 / 10, P_{U}\left(u_{2}\right)=$ $5 / 12$ e $P_{U}\left(u_{3}\right)=17 / 60$.

Segundo o procedimento de codificação para a técnica SHSAS descrito na Seção III-.1 a árvore ilustrada na Figura 2 é obtida. Observa-se, portanto, que as palavras-código associadas aos homofonemas e ao símbolo mudo são $v_{11} \rightarrow$ $00, v_{12} \rightarrow 11100, v_{21} \rightarrow 01, v_{22} \rightarrow 110, v_{23} \rightarrow 11101, v_{31} \rightarrow$ $10, v_{32} \rightarrow 11110$ e $\Delta \rightarrow 1111$.

Os homofonemas correspondentes ao símbolo $u_{1}$ são escolhidos dentre os elementos pertencentes 


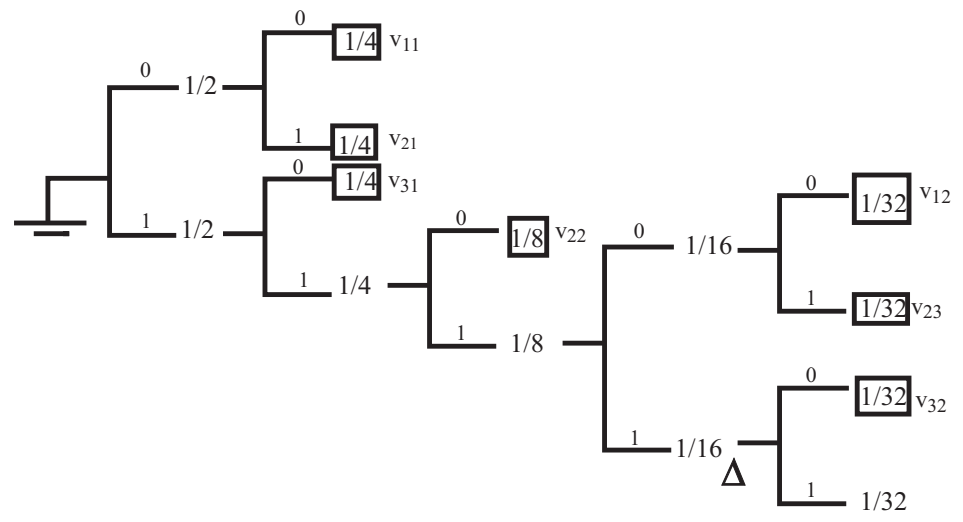

Fig. 2. Árvore obtida para a fonte $U$ com distribuição de probabilidade $P_{U}\left(u_{1}\right)=3 / 10, P_{U}\left(u_{2}\right)=5 / 12$ e $P_{U}\left(u_{3}\right)=17 / 60$ ao se utilizar a técnica de substituição homofônica símbolo-a-símbolo.

aos subconjuntos $V_{11}=\left\{v_{11}, \Delta v_{11}, \Delta \Delta v_{11}, \ldots\right\} \quad \mathrm{e}$ $V_{12}=\left\{v_{12}, \Delta v_{12}, \Delta \Delta v_{12}, \ldots\right\}, \quad$ os homofonemas correspondentes a $u_{2}$ são selecionados nos subconjuntos $V_{21}=\left\{v_{21}\right\}, \quad V_{22}=\left\{v_{22}, \Delta v_{22}, \Delta \Delta v_{22}, \ldots\right\}$ e $V_{23}=\left\{v_{23}, \Delta v_{23}, \Delta \Delta v_{23}, \ldots\right\}$, enquanto que os homofonemas relacionados a $u_{3}$ são selecionados nos subconjuntos, $V_{31}=\left\{v_{31}\right\}, V_{32}=\left\{v_{32}, \Delta v_{32}, \Delta \Delta v_{32}, \ldots\right\}$. Logo, o código resultante para a fonte $V$ é representado por $\left\{(1111)^{i} 00,(1111)^{i} 11100,01,(1111)^{i} 110,(1111)^{i} 11101,10\right.$, $\left.(1111)^{i} 11110\right\}$ e o comprimento médio, calculado a partir de (2), é dado por,

$$
\begin{aligned}
E_{S A S}(W)= & \left(\frac{1}{4}\right) \sum_{r=0}^{\infty}(2+4 r)\left(\frac{1}{16}\right)^{r} \\
& +\left(\frac{1}{32}\right) \sum_{r=0}^{\infty}(5+4 r)\left(\frac{1}{16}\right)^{r} \\
& +\left(\frac{1}{4}\right) \cdot 2+\left(\frac{1}{8}\right) \sum_{r=0}^{\infty}(3+4 r)\left(\frac{1}{16}\right)^{r} \\
& +\left(\frac{1}{32}\right) \sum_{r=0}^{\infty}(5+4 r)\left(\frac{1}{16}\right)^{r} \\
& +\left(\frac{1}{4}\right) \cdot 2+\left(\frac{1}{32}\right) \sum_{r=0}^{\infty}(5+4 r)\left(\frac{1}{16}\right)^{r} \\
& =2,5667 .
\end{aligned}
$$

Como, a entropia da fonte deste exemplo é $H(U)=$ $(3 / 10) \log (10 / 3)+(5 / 12) \log (12 / 5)+(17 / 60) \log (60 / 17)=$ 1,5629 , então pela Definição 2 , observa-se que a eficiência obtida pela técnica SH-SAS neste exemplo é $\eta_{S A S}=0,6089$.

Abordando as técnicas JKM modificada e RM para a mesma fonte.

- JKM modificada

Como visto anteriormente as decomposições das probabilidades dos símbolos $u_{1}, u_{2}$ e $u_{3}$, são dadas por (12), (13) e (14), respectivamente.

De um modo geral, na técnica JKM modificada [4], após a decomposição das probabilidades dos símbolos da fonte são identificados os componentes periódicos e não-periódicos das decomposições das probabilidades dos símbolos da fonte. Os termos não-periódicos e os primeiros termos fornecem as probabilidades dos símbolos homofonemas, enquanto que as razões fornecem as probabilidades dos símbolos mudos. A partir daí é construído um código $D$-ário livre de prefixo.

Se todas as razões são iguais, um único símbolo mudo é usado. Note, que neste exemplo, a decomposição de $u_{1}$ e a decomposição de $u_{3}$ tem mesma razão, $1 / 16$, no entanto, a decomposição de $u_{2}$ apresenta razão distinta, 1/4. Logo, para esta fonte ao se utilizar a técnica JKM modificada são usados dois símbolos mudo, $\Delta_{1}$ e $\Delta_{2}$, com probabilidades, $P\left(\Delta_{1}\right)=1 / 16$ e $P\left(\Delta_{2}\right)=1 / 4$, respectivamente.

Cada termo não-periódico corresponde a um subconjunto com um único homofonema e cada elemento periódico corresponde a um subconjunto com número infinito contável de homofonemas.

Desta forma, nota-se que o símbolo $u_{1}$ tem seus homofonemas nos subconjuntos $V_{11}=\left\{v_{11}, \Delta_{1} v_{11}, \Delta_{1} \Delta_{1} v_{11}, \ldots\right\}$ e $V_{12}=$ $\left\{v_{12}, \Delta v_{12}, \Delta_{1} \Delta_{1} v_{12}, \ldots\right\}, \quad u_{2} \quad$ nos subconjuntos $V_{21}=\left\{v_{21}\right\}$ e $V_{22}=\left\{v_{22}, \Delta_{2} v_{22}, \Delta_{2} \Delta_{2} v_{22}, \ldots\right\}$ e $u_{3}$ nos subconjuntos, $V_{31}=\left\{v_{31}\right\}$ e $V_{32}=\left\{v_{32}, \Delta_{2} v_{32}, \Delta_{2} \Delta_{2} v_{32}, \ldots\right\}$ onde $v_{11} \rightarrow$ $10, v_{12} \rightarrow 11110, v_{21} \rightarrow 00, v_{22} \rightarrow 110, v_{31} \rightarrow$ $01, v_{32} \rightarrow 11111, \Delta_{1} \rightarrow 1110$ e $\Delta_{2} \rightarrow 11$. Logo, o código resultante para a fonte $V$ é representado por $\left\{(1110)^{i} 10,(1110)^{i} 11110,00,(11)^{i} 110,01,(1110)^{i} 11111\right\}$ e o comprimento médio,

$$
\begin{aligned}
E_{J K M}(W)= & \left(\frac{1}{4}\right) \sum_{r=0}^{\infty}(2+4 r)\left(\frac{1}{16}\right)^{r} \\
& +\left(\frac{1}{32}\right) \sum_{r=0}^{\infty}(5+4 r)\left(\frac{1}{16}\right)^{r} \\
& +\left(\frac{1}{4}\right) \cdot 2+\left(\frac{1}{8}\right) \sum_{r=0}^{\infty}(3+2 r)\left(\frac{1}{4}\right)^{r} \\
& +\left(\frac{1}{4}\right) \cdot 2+\left(\frac{1}{32}\right) \sum_{r=0}^{\infty}(5+4 r)\left(\frac{1}{16}\right)^{r} \\
& =2,5667,
\end{aligned}
$$

e portanto, a eficiência, $\eta_{J K M}=\frac{H(U)}{E_{J K M}(W)}=$ $\frac{1,5629}{2,5667} \Rightarrow \eta_{J K M}=0,6089$.

- RM

Pela técnica RM [7] o símbolo mudo é selecionado com probabilidade $P(\Delta)=1 / 16$ e a fonte $U$ é selecionada com probabilidade $15 / 16$, desta forma a fonte expandida tem distribuição de probabilidade dada por $\{1 / 4,1 / 32,1 / 4,1 / 8,1 / 64,1 / 4,1 / 64,1 / 16\}$ (Figura 3). Assim, o comprimento médio é dado por 


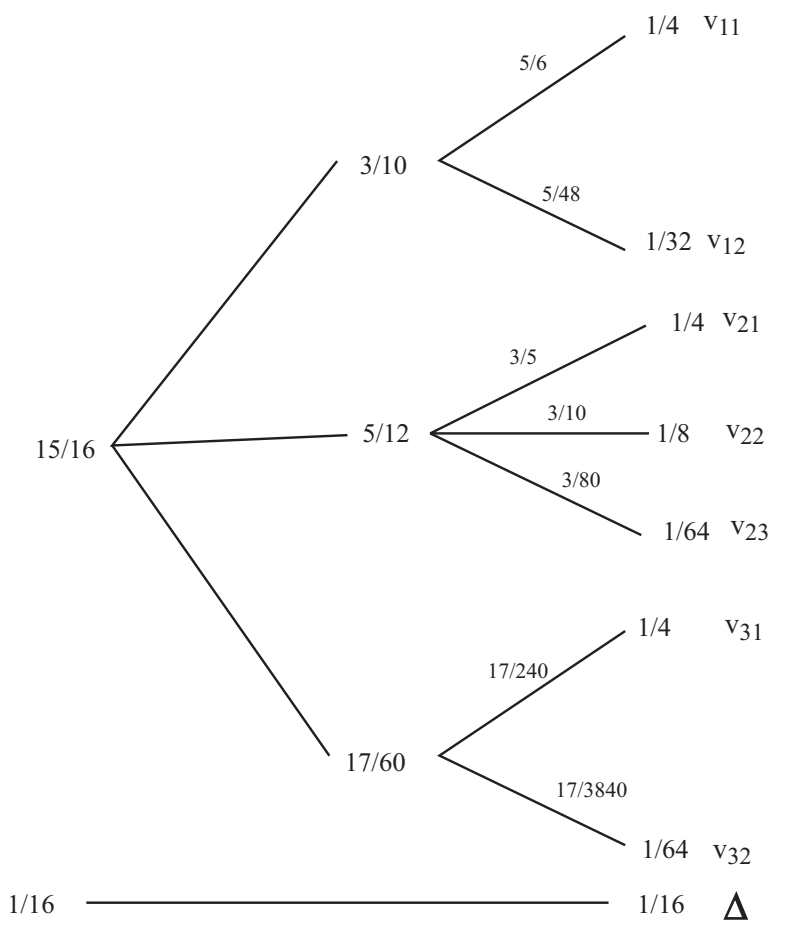

Fig. 3. Técnica RM para a fonte $U$ com distribuição de probabilidade $P_{U}\left(u_{1}\right)=3 / 10, P_{U}\left(u_{2}\right)=5 / 12$ e $P_{U}\left(u_{3}\right)=17 / 60$.

$$
\begin{aligned}
E_{R M}(W)= & \left(\frac{1}{4}\right) \cdot 2+\left(\frac{1}{32}\right) \cdot 5+\left(\frac{1}{4}\right) \cdot 2 \\
& +\left(\frac{1}{8}\right) \cdot 3+\left(\frac{1}{64}\right) \cdot 6+\left(\frac{1}{4}\right) \cdot 2 \\
& +\left(\frac{1}{64}\right) \cdot 6+\left(\frac{1}{16}\right) \cdot 4=2,4688
\end{aligned}
$$$$
\text { sendo a eficiência dada por } \eta_{R M}=\frac{(1-P(\Delta)) H(U)}{E_{R M}(W)}=
$$$$
\frac{\left(\frac{15}{16}\right) 1,5629}{2,4688} \Rightarrow \eta_{R M}=0,5935 \text {. }
$$

\section{Conclusões}

A técnica SH-SAS apresenta a mesma eficiência que é obtida pela técnica JKM modificada. Em particular, quando a decomposição das probabilidades dos símbolos da fonte apresenta a mesma razão, comum a todos os símbolos, as probabilidades dos homofonemas coincidem nos dois casos, assim como também coincidem as probabilidades dos símbolos mudos. Neste caso, as técnicas JKM modificada e SH-SAS apresentam mesmo conjunto de homofonemas, uma vez que ambas as técnicas constroem cada palavra-código homofônica como a concatenação de palavras-código mais curtas derivadas a partir das probabilidades da fonte.

Na técnica JKM modificada [4], a razão de cada série geométrica infinita, produzida pela decomposição das probabilidades de determinados símbolos da fonte, é associada a um símbolo mudo, sendo as razões iguais mapeadas para um mesmo símbolo mudo. Portanto, caso haja razões distintas, haverá símbolos mudos distintos. A técnica SH-SAS utiliza apenas um único símbolo mudo, ao qual corresponde uma única palavra-código, que precisará ser detectada e apagada durante a decodificação (vide exemplo 2).

Ao se analisar a técnica RM percebe-se que, caso a decomposição das probabilidades dos símbolos da fonte apresente apenas termos periódicos, a eficiência obtida é a mesma para as três técnicas (RM, JKM e SH-SAS). Porém, quando a decomposição das probabilidades dos símbolos da fonte apresenta termos não-periódicos, a técnica RM apresenta uma eficiência menor que as demais.

\section{AGRADECIMENTOS}

Este trabalho recebeu apoio parcial do Conselho Nacional de Desenvolvimento Científico e Tecnológico - CNPq, através dos projetos 141215/2002-0 e 305226/2003-7.

\section{REFERÊNCIAS}

[1] H. N. Jendal, Y. J. B. Kuhn and J. L. Massey, "An Information-Theoretic Approach to Homophonic Substitution", Advances in CryptologyEurocrypt'89 (Eds. J.-J. Quisquater and J. Vandewalle), LNCS No. 434. Springer, pp. 382-394, 1990.

[2] C. E. Shannon, "Communication theory of secrecy systems", Bell System Tech. J., vol.28, pp. 656-715, Oct. 1949.

[3] Ch. G. Günther, "A Universal Algorithm for Homophonic Coding", Advances in Cryptology- Eurocrypt'88, Lecture Notes in Computer Science, No.330. Heidelberg and New York: Springer, pp. 405-414, 1988.

[4] V. C. da Rocha, "Perfect homophonic substitution with finite memory", Proc. IEEE International Symposium on Information Theory, 30 June 05 July 2002, Lausanne, Switzerland, p. 409.

[5] N. Abramson, Information Theory and Coding. McGraw-Hill, 1963.

[6] V. C. da Rocha Jr., C. Pimentel and D. P. B. A. Camara, "Redundancy in homophonic coding and a new homophonic coding technique", Proceedings of the International Symposium on Information Theory ISIT 2006, Seattle, Washington, pp. 1253-1257, 9-14 July 2006.

[7] V. C. da Rocha and J. L. Massey, "Better than "optimum" homophonic substitution", Proc. IEEE International Symposium on Information Theory, 25-30 June 2000, Sorrento, Italy, p. 241.

[8] V. C. da Rocha, "On the minimum redundancy of homophonic coding", International Telecomunications Symposium - ITS2002, 30 June - 05 July 2002, Natal-RN, Brasil, pp.310-314.

[9] V. C. da Rocha and M.M. Vasconcelos, "Nova geração da representação D-ária de um número racional", XXII Simpósio Brasileiro de Telecomunicações, 2005, Campinas. Anais do XXIII Simpósio Brasileiro de Telecomunicações, 2005, pp. 573-575. 